\title{
Impact of groin flap ischemia-reperfusion on red blood cell micro-rheological parameters in a follow-up study on rats
}

\author{
Zsuzsanna Magyar, Abel Molnar, David Belkin Nachmias, Din Mann, Viktoria Sogor, \\ Anita Mester, Katalin Peto and Norbert Nemeth* \\ Department of Operative Techniques and Surgical Research, Faculty of Medicine, University of Debre- \\ cen, Debrecen, Hungary
}

\begin{abstract}
.
BACKGROUND: Flap hypoperfusion or ischemia-reperfusion (I/R) may occur during preparation-transposition procedures and by postoperative thrombotic complications. Behind the microcirculatory disturbances micro-rheological alterations are also supposed.

OBJECTIVE: We aimed to investigate the groin flap I/R with following-up micro-rheological parameters.

METHODS: Anesthetized rats were subjected to Control or I/R groups. Groin flaps were prepared bilaterally, pedicled on the superficial epigastric vessels. In Control group the flaps were re-sutured after one hour, while in I/R group microvascular clips were applied on the pedicles for 60 minutes, then the flaps were repositioned. Besides daily wound control, before the operation and on the $1 \mathrm{st}, 3 \mathrm{rd}, 5 \mathrm{th}, 7$ th and 14th postoperative days blood samples were collected for testing red blood cell (RBC) deformability (rotational ektacytometry) and aggregation (light-transmission aggregometry).

RESULTS: RBC deformability significantly worsened by the 3rd-7th postoperative day in I/R group. RBC aggregation enhanced significantly by the 1 st day, in I/R group it remained elevated on the 3 rd day as well. In a complicated case with unilateral flap necrosis, RBC deformability and aggregation worsening was outlined from its group (base, 1st, 3rd day).

CONCLUSION: Wound healing affected micro-rheological parameters in the early postoperative period. Flap I/R exacerbated the alterations. The parameters markedly worsened in case of flap necrosis.
\end{abstract}

Keywords: Ischemia, microcirculation, hemorheology

\section{Introduction}

In the reconstructive surgical procedures various pedicled flaps can be used for covering tissue defects $[6,17,19,20]$. For reconstructive surgery the choice is always depending on the region involved; but the common interest, the most important question of such procedures is the flap survival rate [12, 17]. Based on their composition and blood supply there are multiple variations in flaps. During their preparation, transposition and (auto)transplantation, the flaps may suffer from hypoperfusion and/or ischemia-reperfusion that can influence wound healing.

It is known from the clinical practice, that one of the most crucial factors for transferred free-flap survival is the ischemia-reperfusion $[1,8,12,16,28,30]$. The possible causes for flap ischemia and necrosis include intraoperative and postoperative ones. Most frequently the intraoperative causes lead to complications, when injuring the blood supply during dissection, or creating too much tension on

*Corresponding author: Norbert Nemeth, MD, PhD Department of Operative Techniques and Surgical Research, Institute of Surgery, Faculty of Medicine, University of Debrecen, H-4032 Debrecen, Nagyerdei krt. 98., Hungary. Tel./Fax: +36 52 416 915; E-mail: nemeth@med.unideb.hu. 
the flap, or twisting or kinking the flap pedicle [9, 13, 28]. However, even preoperative anemia (caused by iatrogenic hemodilution and acute blood losses) could significantly impact flap morbidity [13].

From the hemorheological parameters the factors related to red blood cell deformability and red blood cell aggregation have great importance, as these factors play significant role in the microcirculation $[4$, $5,10,18]$. These parameters show changes in numerous pathophysiological conditions, therefore the examination of these parameters is important in surgical and microsurgical experiments as well [18].

We supposed that micro-rheological investigations might be informative in better understanding the pathophysiology of flaps' hypoperfusion and/or ischemia-reperfusion that can be occurred during flap preparation, transposition and transplantation procedures, and in context with the wound healing. Numerous investigations were done in different types of experimental animals to improve surgical safety $[7,15,26,27,32]$. At our Department some studies were completed earlier in this field: using latissimus dorsi muscle flaps on beagle dogs [26], and latissimus dorsi - cutaneous maximus musculocutaneous flaps on rats [15]. In this current study we aimed to investigate this issue on groin adipocutaneous flaps in follow-up study in rats.

\section{Materials and methods}

\subsection{Experimental animals, operative techniques and sampling protocol}

The experiments were approved and registered by the University of Debrecen Committee of Animal Welfare (permission Nr.: 20/2011. UDCAW), in accordance with the relevant Hungarian Animal Protection Act (Law XVIII/1998).

Twenty healthy male CD outbred rats (bodyweight: $399.5 \pm 70.7 \mathrm{~g}$ ) were randomly and equally divided in two experimental groups. All procedures were done under general anesthesia (Thiopental: $60 \mathrm{mg} / \mathrm{kg}$ ). The inguinal regions of the rats were completely shaved, then disinfected with Betadine. In both groups the groin adipocutaneous pedicled flaps -containing the superficial epigastric artery and vein- were prepared bilaterally (area: $8.24 \mathrm{~cm}^{2}$, using a pre-prepared ellipsoid template) (Fig. 1A-C). In the Control group the flaps were repositioned and sutured (4/0 Dexon, 32 single interrupted stitches) into its original bed after one hour. In the $\mathrm{I} / \mathrm{R}$ group the vessels were clamped by microvascular clips for 1 hour (Fig. 1D). After the ischemic period the clips were removed, and the flaps were repositioned and sutured.

Right after the procedure and later on the 1st and 3rd day analgesia was given $(2.5 \mathrm{mg} / \mathrm{kg}$ Flunixin, s.c.). For preventing autophagy, plastic rodents' Elizabethan collars were used during the first 5-7 postoperative days. The animals' behavior was observed and wound inspection was carried out. The postoperative observation period lasted two weeks.

Before the operation, and on the 1st, 3rd, 5th, 7th and 14th postoperative (p.o.) days blood samples were taken from the lateral tail vein (anticoagulant: $\mathrm{K}_{3}$-EDTA $1.5 \mathrm{mg} / \mathrm{ml}$ ) for laboratory measurements.

\subsection{Laboratory methods}

The qualitative and quantitative hematological parameters were determined by Sysmex F-800 microcell counter (TOA Medical Electronics Corp., Ltd., Japan).

The red blood cell aggregation was tested by a Myrenne MA-1 erythrocyte aggregometer (Myrenne $\mathrm{GmbH}$, Germany). The technique is based on light transmittance method [10]. The device determines red blood cell aggregation index values at $0 \mathrm{~s}^{-1}$ (M index) or $3 \mathrm{~s}^{-1}$ shear rates (M1 index), and at the 5th and 10th second, so providing M 5s, M1 5s, M 10s and M1 10s values. The higher index values represent enhanced red blood cell aggregation $[2,11]$. 


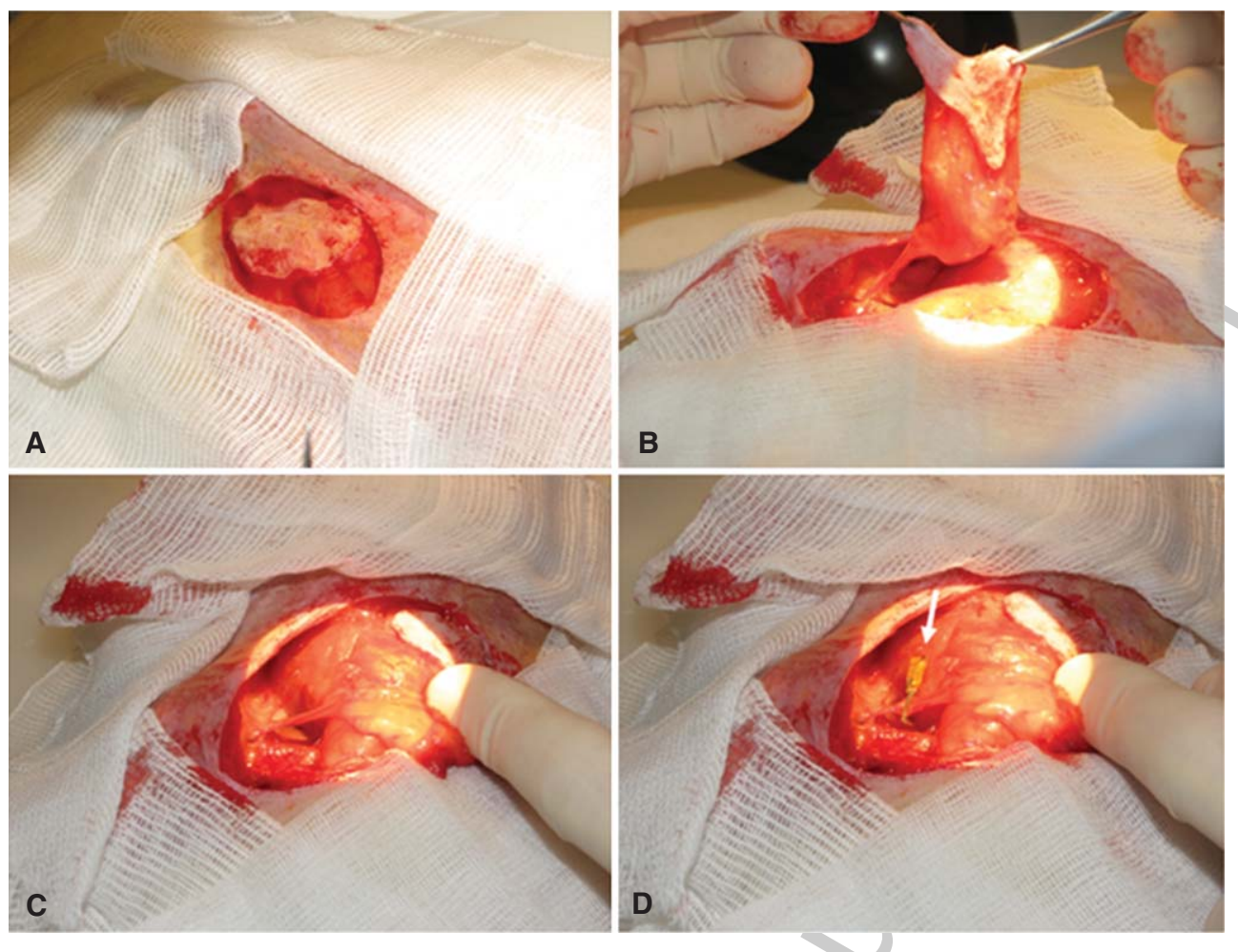

Fig. 1. The preparation of the pedicled groin adipocutaneous flap (A,B), containing the superficial epigastric artery and vein (C), and the clamped pedicle on the groin adipocutaneous flap in the I/R group (D).

Red blood cell deformability was measured using a LoRRca MaxSis Osmoscan ektacytometer (Mechatronics BV, The Netherlands), by which the elongation index (EI) was determined in the function of shear stress (SS, range: $0.5-30 \mathrm{~Pa}$ ) on blood samples suspended in polyvinylpyrrolidone (PVP) phosphate buffered saline (PBS) solution (PVP: $360 \mathrm{kDa}$, Sigma-Aldrich Co. USA; PVP-PBS solution viscosity $=34.2 \mathrm{mPas}$, osmolality $=294 \mathrm{mOsmol} / \mathrm{kg}, \mathrm{pH}=7.1$ ). The higher EI reflects better red blood cell deformability $[2,11]$. Measurements were carried out at $37^{\circ} \mathrm{C}$. For the comparison of the EI-SS curves the Lineweaver-Burk analysis was applied, and the ratio of maximal elongation index $\left(\mathrm{EI}_{\max }\right)$ and the shear stress value at half $\mathrm{EI}_{\max }\left(\mathrm{SS}_{1 / 2}[\mathrm{~Pa}]\right)$ was also used [3].

\subsection{Statistical analysis}

Data are presented as means \pm standard deviation (S.D.). One way and repeated measures ANOVA tests were used for intra- and inter-group comparisons (Bonferroni/Dunn methods). For simple comparison of inter-group differences at single time points, $t$-test/Mann-Whitney rank sum tests were applied as well, depending on the normality of data distribution. A $p<0.05$ value was considered statistically significant.

\section{Results}

\subsection{Hematological parameters}

Total leukocyte count (white blood cell count, WBC [G/l]) showed a moderate increase in the Control group by the 1st postoperative (p.o.) day. Afterwards, both groups expressed significantly 

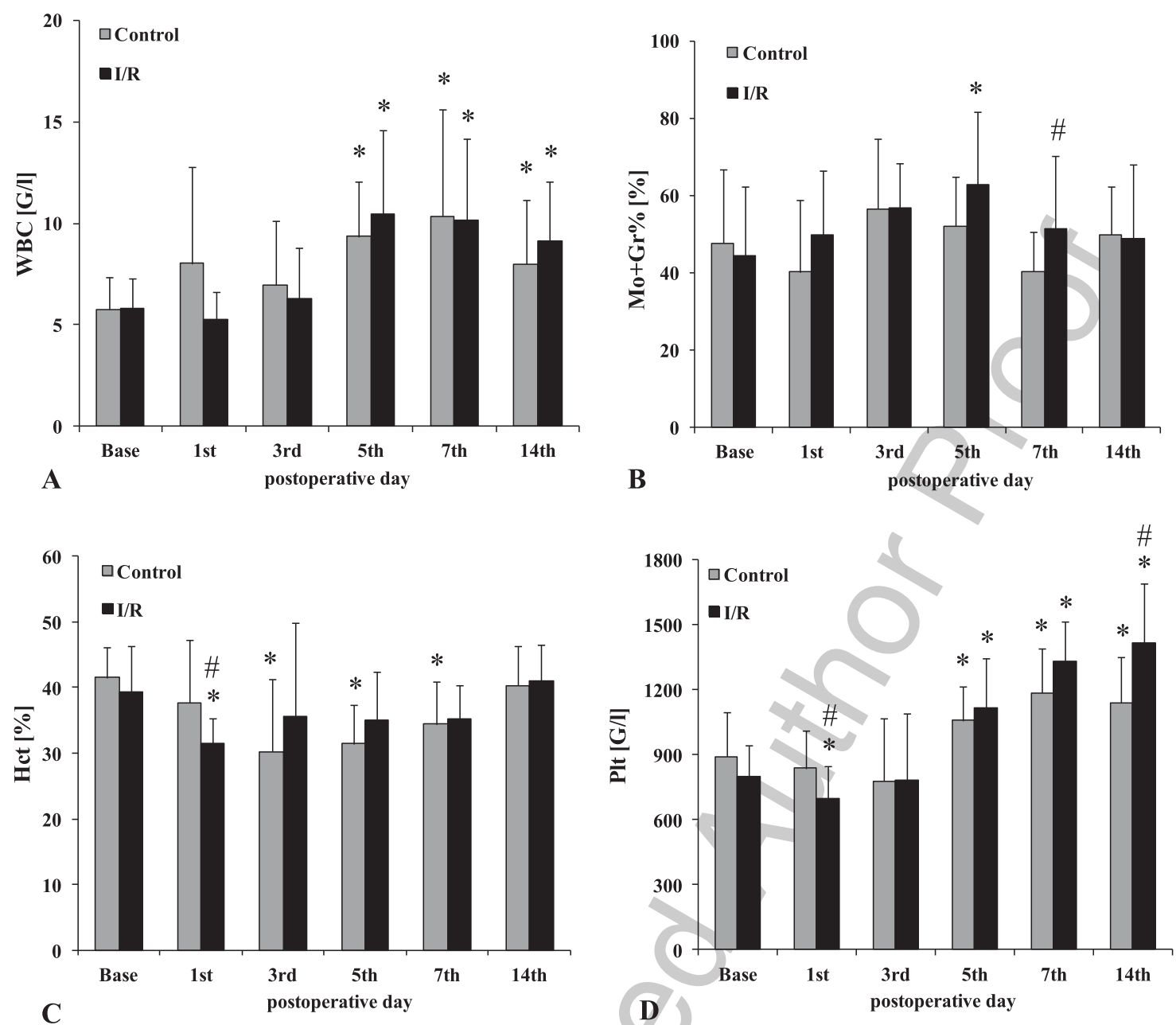

Fig. 2. Alterations of the white blood cell count (WBC [G/l]) (A), the monocyte+granulocyte ratio (Mo+Gr [\%]) (B), the hematocrit (Hct [\%]) (C), and the platelet count (Plt [G/l]) (D) in the Control and Ischemia-Reperfusion (I/R) groups during the two-week postoperative follow-up period. Means \pm S.D.; ${ }^{*} p<0.05$ vs. base; ${ }^{\#} p<0.05$ vs. Control.

elevated WBC count between the 5th-14th p.o. days, as the followings: on the 5th p.o. day $p<0.001$ vs. base in both groups; on the 7th p.o. day in Control group $p=0.002$ vs. base, in I/R group $p<0.001$ vs. Control; and on the 14th day in Control group $p=0.008$, and in I/R group $p<0.001$ compared to base values (Fig. 2A). Monocyte+granulocyte ratio (\%) showed an elevation on the 3rd-5th p.o. days (in I/R group $p=0.007$ vs. base, and $p=0.051$ vs. Control). On the second week of the follow-up period the values normalized (7th day in I/R group: $p=0.047$ vs. Control) (Fig. 2B).

Hematocrit (Hct [\%]) values decreased over the first postoperative week probably due to the regularly blood samplings (1st day: in I/R group $p<0.001$ vs. base and $p=0.031$ vs. Control; 3rd, 5th and 7th days: in Control group $p<0.001$ vs. base). By the 14 th day the values normalized without important difference between the groups (Fig. 2C).

Platelet count (Plt [G/l]), after a decrease in I/R group by the first p.o. day ( $p=0.033$ vs. base and $p=0.009$ vs. Control), showed a gradual increase over the second week of the observation period in both groups. By the 5th postoperative day the magnitude of the increase was almost similar in the groups (Control: $p=0.006$; in I/R group: $p<0.001$ vs. base). On the 7 th day the I/R group's values 

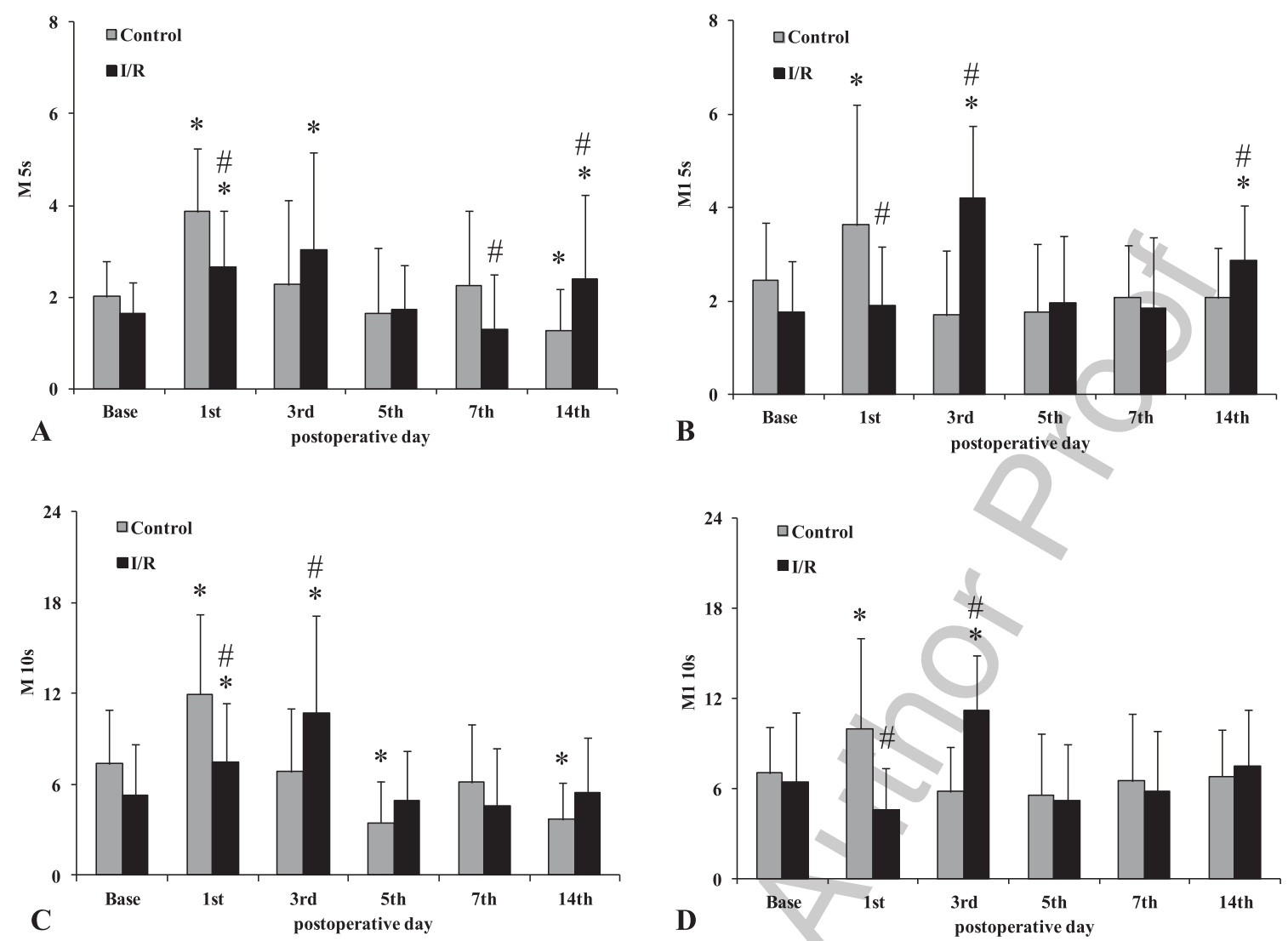

Fig. 3. Changes of the red blood cell aggregation index values (M 5s, M1 5s, M 10s and M1 10s) (A-D) in the Control (A) and Ischemia-Reperfusion (I/R) (B) groups during the two-week postoperative follow-up period. Means \pm S.D.; ${ }^{*} p<0.05$ vs. base; ${ }^{*} p<0.05$ vs. Control.

markedly exceeded the Control values ( $p<0.001$ vs. base in both groups). By the 14th postoperative day the I/R group values increased further ( $p<0.001$ vs. base and $p=0.003$ vs. Control), while Control values were similar to the 7 th day data ( $p<0.001$ vs. base) (Fig. 2D).

\subsection{Red blood cell aggregation}

Figure 3 shows the changes of the four aggregation index parameters determined by the Myrenne aggregometer. In general, aggregation index showed an increase by the 1st and 3rd postoperative days that were followed by lowering values.

The increase by the 1st p.o. day in the Control group was larger in magnitude (M 5s: $p<0.001, \mathrm{M} 1$ 5s: $p=0.011$, M 10s: $p<0.001$ and M1 10s: $p=0.006$ vs. base) compared to the moderate increase in I/R group (M 5s: $p<0.001$ vs. base, M 10s: $p=0.011$ vs. base and $p<0.001$ vs. Control, M1 10s: $p<0.001$ vs. Control). By the 3rd p.o. day the I/R group's values showed rising (M 5s: $p=0.002$ vs. base, M1 5s: $p<0.001$ vs. base and Control, M 10s: $p=0.002$ vs. base and $p=0.001$ vs. Control, M1 10s: $p=0.002$ vs. base and $p<0.001$ vs. Control). The I/R groups' values on the 14 th p.o. day increased over the Control values (M 5s: $p=0.042$ vs. base and $p=0.012$ vs. Control, M1 5s: $p<0.001$ vs. base and $p=0.033$ vs. Control) (Fig. 3). 


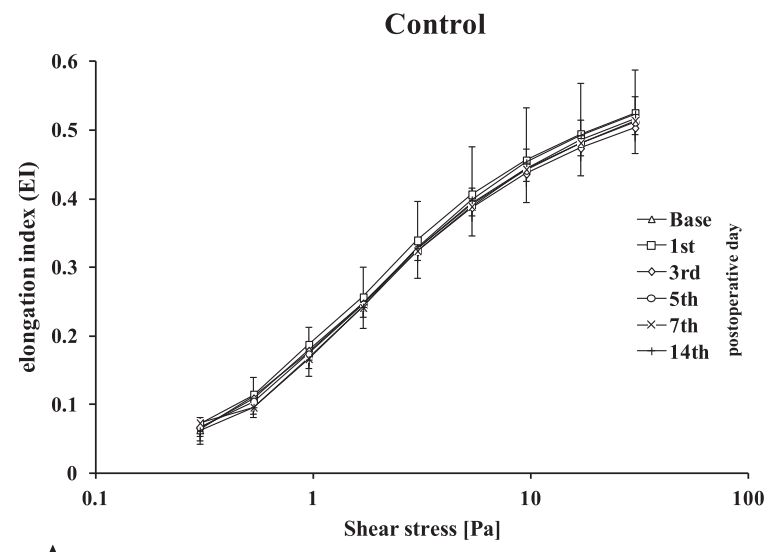

A

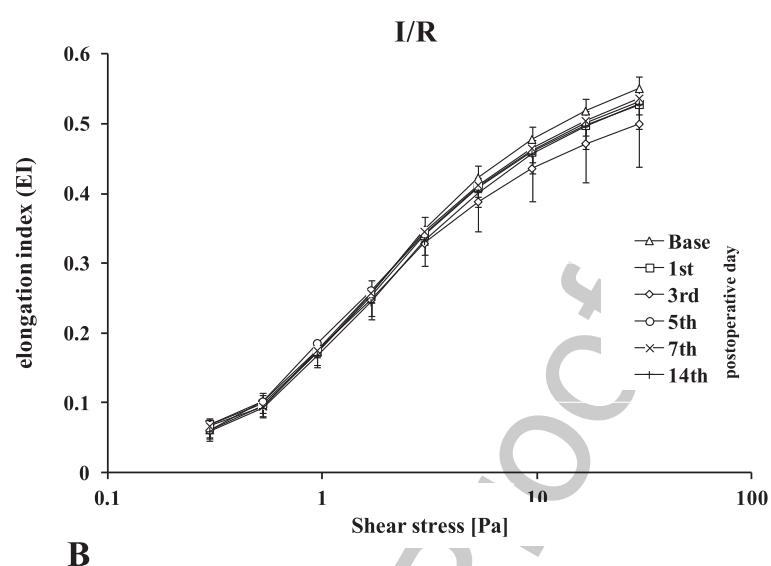

B

Fig. 4. Changes of the red blood cell deformability, as elongation index (EI) in the function of shear stress (SS [Pa]), in the Control (A) and Ischemia-Reperfusion (I/R) (B) groups during the two-week postoperative follow-up period. Means \pm S.D.

Table 1

Red blood cell deformability parameters given by the Ektacytometry measurements: elongation index (EI) at $3 \mathrm{~Pa}$ shear stress (SS), maximal elongation index $\left(\mathrm{EI}_{\max }\right)$ and the shear stress belonging to the half $\mathrm{EI}_{\max }\left(\mathrm{SS}_{1 / 2}\right)$, as well as their ratio

\begin{tabular}{|c|c|c|c|c|c|c|c|}
\hline \multirow[t]{2}{*}{ Variable } & \multirow[t]{2}{*}{ Group } & \multirow[t]{2}{*}{ Base } & \multicolumn{5}{|c|}{ Postoperative days } \\
\hline & & & $1 \mathrm{st}$ & 3 rd & 5 th & 7 th & 14 th \\
\hline \multirow[t]{2}{*}{ EI at $3 \mathrm{~Pa}$} & Control & $0.326 \pm 0.071$ & $0.339 \pm 0.017$ & $0.322 \pm 0.038$ & $0.326 \pm 0.016$ & $0.324 \pm 0.016$ & $0.328 \pm 0.014$ \\
\hline & $\mathrm{I} / \mathrm{R}$ & $0.348 \pm 0.018$ & $0.339 \pm 0.028$ & $0.329 \pm 0.032^{*}$ & $0.342 \pm 0.012^{\#}$ & $0.345 \pm 0.012^{\#}$ & $0.332 \pm 0.019^{*}$ \\
\hline \multirow[t]{2}{*}{$\mathrm{EI}_{\max }$} & Control & $0.545 \pm 0.093$ & $0.555 \pm 0.015^{*}$ & $0.530 \pm 0.035^{*}$ & $0.542 \pm 0.021$ & $0.545 \pm 0.033$ & $0.562 \pm 0.031$ \\
\hline & $\mathrm{I} / \mathrm{R}$ & $0.593 \pm 0.016^{\#}$ & $0.565 \pm 0.034^{\#}$ & $0.531 \pm 0.071$ & $0.560 \pm 0.018^{\#}$ & $0.569 \pm 0.011^{\#}$ & $0.568 \pm 0.027$ \\
\hline \multirow[t]{2}{*}{$\mathrm{SS}_{1 / 2}[\mathrm{~Pa}]$} & Control & $2.19 \pm 0.58$ & $1.99 \pm 0.26$ & $2 \pm 0.42$ & $2.06 \pm 0.29$ & $2.13 \pm 0.21$ & $2.23 \pm 0.21$ \\
\hline & $\mathrm{I} / \mathrm{R}$ & $2.22 \pm 0.29$ & $2.1 \pm 0.31$ & $1.94 \pm 0.51 *$ & $1.94 \pm 0.15^{*}$ & $2.04 \pm 0.15$ & $2.25 \pm 0.35$ \\
\hline \multirow{2}{*}{$\begin{array}{c}\mathrm{EI}_{\max } / \mathrm{SS}_{1 / 2} \\
{\left[\mathrm{~Pa}^{-1}\right]}\end{array}$} & Control & $0.268 \pm 0.077$ & $0.285 \pm 0.035$ & $0.276 \pm 0.057$ & $0.267 \pm 0.034$ & $0.257 \pm 0.019$ & $0.253 \pm 0.021$ \\
\hline & $\mathrm{I} / \mathrm{R}$ & $0.271 \pm 0.033$ & $0.274 \pm 0.046$ & $0.282 \pm 0.051$ & $0.291 \pm 0.022$ & $0.280 \pm 0.024$ & $0.257 \pm 0.033$ \\
\hline
\end{tabular}

Means \pm S.D., ${ }^{*} p<0.05$ vs. base, ${ }^{\#} p<0.05$ vs. Control.

\subsection{Red blood cell deformability}

We found that red blood cell deformability values were markedly worsened on the 1st-5th postoperative days dominantly in the I/R group (Fig. 4, Table 1).

The decrease of elongation index data (at $3 \mathrm{~Pa}$ ) was significant by the $3 \mathrm{rd}(p=0.002 \mathrm{vs}$. base), the 5 th ( $p=0.007$ vs. Control), the 7 th ( $p<0.001$ vs. Control) and the 14 th $(p=0.018$ vs. base $)$ p.o. days. The calculated $\mathrm{EI}_{\max }$ of the Control group showed an initial increase by the 1 st day ( $p=0.006$ vs. base) and decreased by the $3 \mathrm{rd}$ p.o. day $(p<0.001$ vs base). Meanwhile in the I/R group a decrease was observed on the 1 st ( $p=0.008$ vs. Control) and the 3rd p.o. days. However, on the 5 th and 7 th days $\mathrm{EI}_{\max }$ values were higher than the Control $\left(p=0.027\right.$ and $p=0.007$, respectively). $\mathrm{SS}_{1 / 2}$ values were relatively stable in the Control group except for a moderate decrease by the 1st and 3rd postoperative days. The I/R group's values were significantly lower than the base on the $3 \mathrm{rd}(p=0.05)$ and the 5th $(p=0.009)$ postoperative days. The ratio of these two calculated parameters did not show significant alterations. 


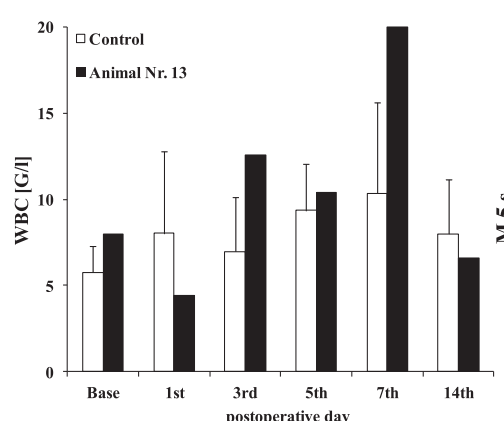

A

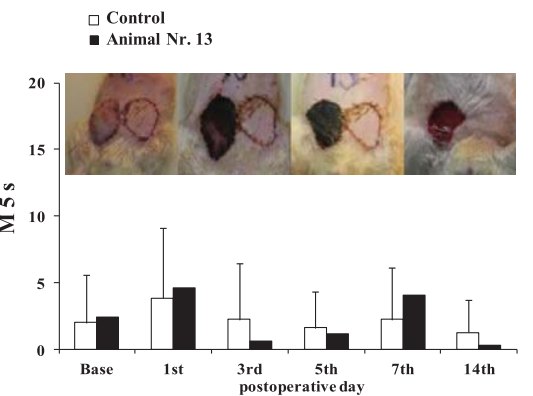

B

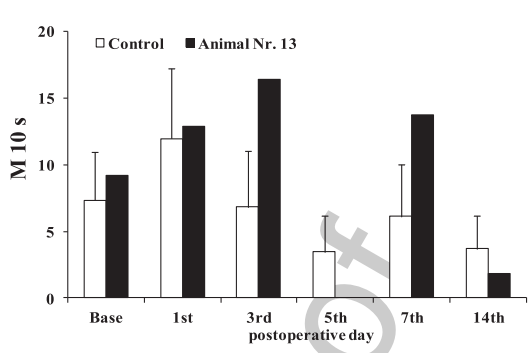

C
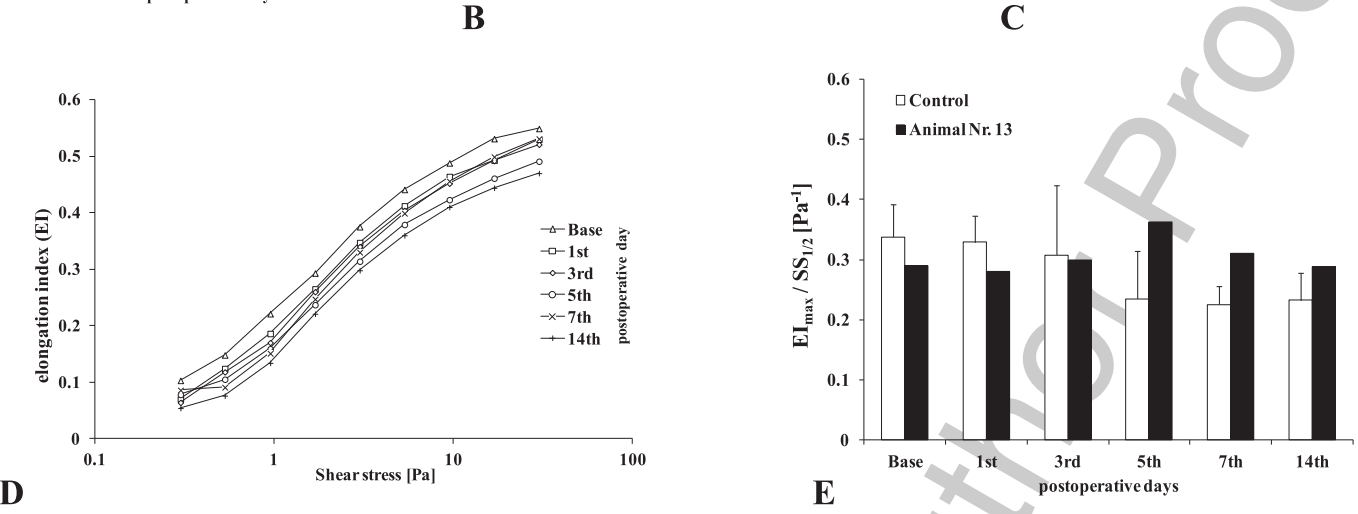

Fig. 5. Comparative values of a case of flap necrosis (animal nr. 13 in Control group), and the values of non-complicated cases within the same group (means \pm S.D.). After flap swelling, thrombosis and necrosis occurred, turning to scar (inserted photos above B). White blood cell count (WBC) (A), red blood cell aggregation (M 5s and M 10s values) (B,C), red blood cell deformability (elongation index in the function of shear stress, and $\mathrm{EI}_{\max } / \mathrm{SS}_{1 / 2}$ values) (D, E).

\subsection{The case of flap necrosis}

In one animal of the Control group (nr. 13) we found a unilateral flap necrosis on the right side. We could see that the flap was livid and edematous already by the 1 st postoperative day. On the 3rd day marginal necrosis was observed at the upper pole of the flap. By the 5th day the extension of the visible necrosis reached about $70 \%$ of the flap, and was completed by the end of the first postoperative week. During the 2nd week the necrotic flap was released revealing a newly formed scar (Fig. 5, inserted photos above the chart B). In this case markedly increased white blood cell count was measured compared to the other animals in the same group (Fig. 5A). The hemoglobin and the hematocrit values were also higher than in the other animals of the group, and the highest platelet value was found in this animal. The aggregation index values were higher in the first week of the postoperative period (Fig. 5B, C). Deformability values markedly worsened on the 5 th and 14 th postoperative days, showing gradual impairment, as it was reflected by the parametric data, as well (Fig. 5D, E).

\section{Discussion}

During wound healing the microcirculation has an important role determining the viability of the flaps [23]. As Kusza and Siemionow worded in their review: "... the knowledge of differences in microcirculatory responses presented by different tissue types should be of interest to microsurgeons and others specialists dealing with tissue ischemia and reperfusion injury to improve outcomes in patients exposed to lengthy procedures and unfavorable perioperative conditions." [18]. 


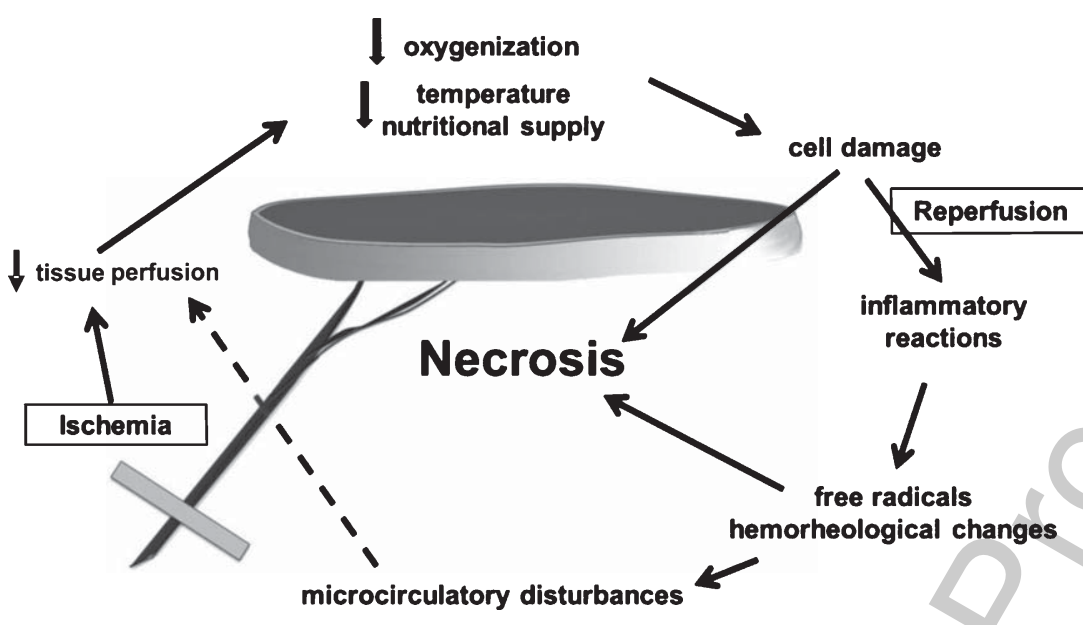

Fig. 6. Overviewing the factors leading to flap necrosis, and the possible vicious circle of hemorheological and microcirculatory disturbances (source: Department of Operative Techniques and Surgical Research, contributor: Dr. Zoltan Klarik).

The viability of the tissues fundamentally depends on the adequate blood supply, which provides the oxygenation and metabolic needs $[20,23,28]$. The cessation of oxygen and nutrition supply caused by the ischemia leads to cell damage. During reperfusion, besides the cellular hypoxic damage, inflammatory reactions and free radical release lead to further tissue damage, resulting in hemorheological disturbances among others $[5,8,31,33]$. The micro-rheological differences can lead to further microcirculatory disturbances that cause decreased tissue perfusion again $[5,18,28,30]$ (Fig. 6).

Many agents and maneuvers have been used to prevent flap ischemia. For instance, as an interesting example, Sen et al. used omeprazol in their study and they found that the increasing gastrin during flap surgeries can be thought as a positive contributor to increase flap viability [25]. Wallmichrath et al. demonstrated that heparin and recombinant tissue plasminogen activator can be protective against flap failure in a rat model using adipocutaneous free flaps [29]. Numerous factors can lead to free flap failure, but the vessel-related accidents are the most important ones, thus, lots of intra- and postoperative methods have been developed to prevent these complications. By way of example, Park et al. studied the effects of a "preoperative treatment" using botulinum toxin B that could enhance the velocity and blood flow of vascular pedicles and decrease vascular accidents [21]. Also, transit-time flow volume measurement, a non-Doppler-based ultrasound technology, could be very useful for the surgeon's decision making in microvascular free tissue transfer procedures, identifying flawed anastomoses and higher flow concomitant veins [24]. Furthermore, laser Doppler fluxmetry, intravital videomicroscopy techniques as well as contrast enhanced ultrasound methods provide useful information about flaps' microvasculature $[9,14,29]$.

We hypothesized that micro-rheological investigations might be informative in understanding better the pathophysiology of flaps' hypoperfusion and/or ischemia-reperfusion. In laboratory rats numerous flap models exist for using them as training models or for experimental microsurgery studying flap pathophysiology $[7,22,27,32]$. In this study we examined groin adipocutaneous flap model, pedicled to the superficial epigastric artery and vein [32].

The hematological and hemorheological "signs" of the acute phase reactions could be observed in both groups, but with different manner and dynamics. Anesthesia, immobilization, tissue preparation, wound healing; all contribute to the presence of acute phase reactions. Concerning the inflammatory reactions, it is important to mention that in all animals about $21-22 \mathrm{~cm}$ wound line was under healing process (two ellipsoid flaps with about $10.7 \mathrm{~cm}$ perimeter per each). The previously 
ischemically damaged flaps triggered more extended inflammatory reaction, leading to the hematological and micro-rheological alterations due to the activated leukocytes, acute phase reactions, that are known to cause impaired red blood cell deformability and/or enhanced red blood cell aggregation $[1,5,8,26]$.

In an earlier canine study, significant red blood cell aggregation enhancement and increased hematocrit were observed in the first hour of the reperfusion of the ischemically insulted (1-hour) latissimus dorsi musculocutaneous flaps testing blood samples taken from the pedicle vein (thoracodorsal vein) [26]. These alterations were not observable in the blood drained from intact flaps, which were without ischemia. The results also showed that the investigations of carbonyl content, TBARS concentration, and GSH content reflected the oxidative damage during reperfusion. On the 7th postoperative day the $\mathrm{I} / \mathrm{R}$ insulted flap was macroscopically more indurated than the control side [26].

Klarik et al. examined the hemorheological (red blood cell deformability and aggregation) and microcirculatory parameters (laser Doppler fluxmetry) after two-hour ischemia in latissimus dorsi cutaneus maximus musculocutaneous flap in a rat model [15]. They found that besides the non-specific, acute phase-driven micro-rheological alterations, the I/R caused deterioration in flap microcirculation. Microcirculatory follow-up was important in prediction the possible postoperative complications, such as flap thrombosis and consequent flap failure [15].

These studies, together with the current one, may provide the summary that ischemia-reperfusion have different effect on various flaps. It is depending on tissue ischemic tolerance. Micro-rheological parameters may provide useful information in flap studies. Intraoperative laser Doppler measurement can be informative in predicting possible postoperative complications, such as thrombosis and/or necrosis $[14,15]$.

In current study we also faced flap failure. The possible reason of the unilateral flap necrosis in animal Nr. 13 could be a thrombotic event during the early postoperative period, probably due to the torsion of the pedicle vessels [20]. Interestingly, this experimental animal was in the Control Group, where microvascular clip was not applied. We suppose that the vessel torsion or kinking could be happened during the reposition. The laboratory parameters changed in parallel with the process (swelling, marginal than extended necrosis, released necrotic tissues and scar formation). The explanation for these results could be the inflammatory reactions, as the necrosis was consummated during the follow-up period.

\section{Conclusion}

As conclusion, the effect of the operation and the wound healing of the flap sutures affected microrheological parameters in the early postoperative period in this groin flap ischemia-reperfusion model. Ischemia and reperfusion exacerbated the alterations mostly on the 3 rd -5 th p.o. days. These parameters were markedly worsened in the complicated case (i.e. flap necrosis). As micro-rheological parameters determine microcirculation as well, their monitoring can be informative in further flap studies.

\section{Acknowledgments}

The authors are grateful for the technical staff of the Department of Operative Techniques and Surgical Research, Faculty of Medicine, University of Debrecen.

The authors comply with the Ethical Guidelines for Publication in Clinical Hemorheology and Microcirculation as published on the IOS Press website and in Volume 63, 2016, pp. 1-2. of this journal. 


\section{References}

[1] R. Anaya-Prado, L.H. Toledo-Pereyra, A.B. Lentsch and P.A. Ward, Ischemia/reperfusion injury, J Surg Res 105(2) (2002), 248-258.

[2] O.K. Baskurt, M. Boynard, G.C. Cokelet, P. Connes, B.M. Cooke, S. Forconi, et al., New guidelines for hemorheological laboratory techniques, Clin Hemorheol Microcirc 42(2) (2009), 75-97.

[3] O.K. Baskurt, M.R. Hardeman, M. Uyuklu, P. Ulker, M. Cengiz, N. Nemeth, et al., Parameterization of red blood cell elongation index-shear stress curves obtained by ektacytometry, Scand J Clin Lab Invest 69(7) (2009), $777-788$.

[4] O.K. Baskurt and H.J. Meiselman, Blood rheology and hemodynamics, Semin Thromb Hemost 29(5) (2003), $435-450$.

[5] O.K. Baskurt, Mechanism of blood rheology alterations, In Baskurt OK, Hardeman MR, Rampling MW, Meiselman HJ, editors. Handbook of Hemorheology and Hemodynamics. Amsterdam: IOS Press; 2007, pp. 170-190.

[6] J.S. Brown, J.-C. Devine, P. Magennis, P. Sillifant, S.N. Rogers and E.D. Vaughan, Factors that influence the outcome of salvage in free tissue transfer, Br J Oral Maxillofac Surg 41(1) (2003), 16-20.

[7] B.C. Cooley and P.M. Werker, Review article of rat muscle and myocutaneous flap models, J Reconstr Microsurg 11(1) (1995), 83-85.

[8] H. Eltzschig and T. Eckle, Ischemia and reperfusion - from mechanism to translation, Nat Med 17(11) (2011), 1391-1401.

[9] S. Geis, L. Prantl, M. Schoeneich, P. Lamby, S. Klein, J. Dolderer, et al., Contrast enhanced ultrasound (CEUS) - an unique monitoring technique to assess microvascularization after buried flap transplantation, Clin Hemorheol Microcirc 62(3) (2016), 205-214.

[10] M. Grau, A. Kollikowski and W. Bloch, Remote ischemia preconditioning increases red blood cell deformability through red blood cell-nitric oxide synthase activation, Clin Hemorheol Microcirc 63(3) (2016), 185-197.

[11] M.R. Hardeman, P.T. Goedhart and S. Shin, Methods in hemorheology. In: Baskurt OK, Hardeman MR, Rampling MW, Meiselman HJ, editorss. Handbook of Hemorheology and Hemodynamics. Amsterdam: IOS Press; 2007, pp. $242-266$.

[12] D.A. Hidalgo, J.J. Disa, P.G. Cordeiro and Q.Y. Hu, A review of 716 consecutive free flaps for oncologic surgical defects: Refinement in donor site selection and technique, Plast Reconstr Surg 102(3) (1998), 722-732.

[13] J.B. Hill, A. Patel, G.A. Del Corral, K.W. Sexton, J.M. Ehrenfeld, O.D. Guillamondegui, et al., Preoperative anemia predicts thrombosis and free flap failure in microvascular reconstruction, Ann Plast Surg 69(4) (2012), 364-367.

[14] F. Jung, B. Leithäuser, H. Landgraf, M. Jünger, U. Franzeck, A. Pries, et al., Laser doppler flux measurement for the assessment of cutaneous microcirculation - critical remarks, Clin Hemorheol Microcirc 55(4) (2013), 411-416.

[15] Z. Klarik, R. Tamas, E. Toth, F. Kiss, E.L. Kovacs, M. Jackel, et al., Intra and postoperativ evaluations of microcirculation and micro-rheological parameters in a rat model of musculocutaneous flap ischemia-reperfusion, Acta Cir Bras 30(8) (2015), 551-560.

[16] J.V. Kozarski, Some biological characteristics of transferred free flaps, Microsurgery 27(5) (2007), 360-368.

[17] A.L. Kruse, H.T. Luebbers, K.W. Grätz and J.A. Obwegeser, Factors influencing survival of free-flap in reconstruction for cancer of the head and neck: A literature review, Microsurgery 30(3) (2010), 242-248.

[18] K. Kusza and M. Siemionow, Is the knowledge on tissue microcirculation important for microsurgeon? Microsurgery 31(7) (2011), 572-579.

[19] S. Miyamoto, S. Kayano, H. Umezawa, M. Fujiki, J. Nakao and M. Sakuraba, Efficient design of a latissimus dorsi musculocutaneous flap to repair large skin defects of the upper back, Microsurgery 34(1) (2014), 20-22.

[20] B.M. O’Brien and W.A. Morrison, Reconstructive microsurgery. Edinburgh, New York: Churchill Livingstone; 1987.

[21] B.Y. Park, H.K. Kim, W.S. Kim and T.H. Bae, The effect of botulinum toxin B pretreatment to the blood flow in the microvascular anastomosis, Ann Plast Surg 72(2) (2014), 214-219.

[22] J.J. Petry and K.A. Wortham, The anatomy of the epigastric flap in the experimental rat, Plast Reconstr Surg 74(3) (1984), 410-413.

[23] C.J. Salgado, S.L. Moran and S. Mardini, Flap monitoring and patient management, Plast Reconstr Surg 124(6 Suppl) (2009), e295-e302.

[24] J.C. Selber, P.B. Garvey, M.W. Clemens, E.I. Chang, H. Zhang and M.M. Hanasono, A prospective study of transit-time flow volume measurement for intraoperative evaluation and optimization of free flaps, Plast Reconstr Surg 131(2) (2013), 270-281.

[25] H. Sen, M. Oruc, V.M. Isik, M. Sadic, H. Sayar, R. Citil, et al., The effect of omeprazole usage on the viability of random pattern skin flaps in rats, Ann Plast Surg (2016). [Epub ahead of print] doi: 10.1097/SAP.0000000000000922

[26] R. Tamas, N. Nemeth, E. Brath, M. Sasvari, C.S. Nyakas, B. Debreczeni, et al., Hemorheological, morphological, and oxidative changes during ischemia-reperfusion of latissimus dorsi muscle flaps in a canine model, Microsurgery 30(4) (2010), 282-288. 
[27] A. Tilgner, U. Herrberger and P. Oswald, Myocutaneous flap models in the rat, Anatomy, histology and operative technique of the latissimus dorsi myocutaneous flap, Z Versuchstierkd 31(5) (1988), 225-232.

[28] M.G. van den Heuvel, W.A. Buurman, A. Bast and R.R. van der Hulst, Review: Ischaemia-reperfusion injury in flap surgery, J Plast Reconstr Aesthet Surg 62(6) (2009), 721-726.

[29] J. Wallmichrath, C. Birk, R.G. Baumeister, T.O. Engelhardt, R.E. Giunta and A. Frick, Examination of the protective effects of heparin and recombinant tissue plasminogen activator (rtPA) in compromised adipocutaneous free flaps in the rat model using intravital video microscopy, Clin Hemorheol Microcirc 59(3) (2015), 185-195.

[30] W.Z. Wang, Investigation of reperfusion injury and ischemic preconditioning in microsurgery, Microsurgery 29(1) (2009), 72-79.

[31] D.M. Yellon and D.J. Hausenloy, Myocardial reperfusion injury, N Engl J Med 357(11) (2007), 1121-1135.

[32] F. Zhang, W.D. Sones and W.C. Lineaweaver, Microsurgical flap models in the rat, J Reconstr Microsurg 17(3) (2001), 211-221.

[33] Y. Zhang, H. Xu, T. Wang, J. He, J. Wei, T. Wang, et al., Remote limb ischemic post-conditioning attenuates ischemiareperfusion injury in rat skin flap by limiting oxidative stress, Acta Cir Bras 31(1) (2016), 15-21. 